UVX 2008 (2009) 157-161

(C) EDP Sciences, 2009

DOI: $10.1051 / \mathrm{uvx} / 2009025$

\title{
Cohérence temporelle et largeur spectrale des lasers XUV transitoires pompés en incidence rasante
}

\author{
J. Habib ${ }^{1,2}$, A. Klisnick ${ }^{1}$, O. Guilbaud ${ }^{1,2}$, D. Joyeux ${ }^{3}$, B. Zielbauer ${ }^{1,2}$, \\ S. Kazamias ${ }^{1,2}$, M. Pittman ${ }^{1,2}$, D. Ros ${ }^{1,2}$ et F. de Dortan ${ }^{1}$ \\ ${ }^{1}$ LIXAM, Laboratoire d'Interaction du Rayonnement $X$ avec la Matière, UMR 8624, \\ Bât. 350, Campus de l'Université Paris XI, 91405 Orsay Cedex, France \\ ${ }^{2}$ LASERIX, Centre Laser Université Paris-Sud, Campus de l'Université Paris XI, \\ 91405 Orsay Cedex, France \\ ${ }^{3}$ Laboratoire Charles Fabry, Institut d'Optique Campus de Polytechnique RD 128, \\ 91127 Palaiseau Cedex, France
}

\begin{abstract}
Résumé. We have used a variable path-difference, wavefront-division interferometer to measure the temporal coherence of an unseeded Ni-like Mo GRIP X-ray laser. This quantity is inversely related to its spectral linewidth. We have investigated the role of several pump parameters on the inferred spectral width. Along this experiment, particular attention has been paid on the source stability.
\end{abstract}

\section{INTRODUCTION}

Des progrès remarquables ont été accomplis ces dernières années pour faire des lasers XUV des sources à haute cadence, intenses et totalement cohérentes. L'injection d'harmoniques laser d'ordre éleve $\mathrm{HOH}$ dans un laser $\mathrm{X}$ constitue l'un des progrès les plus significatifs obtenus [1]. Plusieurs points restent toutefois à améliorer pour que le laser $\mathrm{X}$ rivalise avec les harmoniques d'ordre élevé ou les lasers $\mathrm{X}$ à électrons libres (XFEL). Si en régime injecté, la durée des impulsions laser $\mathrm{X}$ atteint maintenant la limite de Fourier [3], elle reste encore dans le domaine picoseconde. Ceci est du à la faible largeur spectrale de l'étage amplificateur laser $\mathrm{X}$ employé. Le rayonnement harmonique possède un spectre beaucoup plus large que le laser XUV. En injectant celui-ci dans le plasma laser XUV, son spectre va subir un rétrécissement, qui entrainera un étirement temporel de l'impulsion au cours de son amplification.

Les lasers XUV générés dans des plasmas de cibles solides présentent potentiellement des largeurs spectrales élevées car la température ionique et les taux de collisions peuvent y devenir importants. C'est pourquoi nous avons mené une étude de la largeur spectrale d'un laser XUV à Molybdène nickelloïde $(\lambda=18.9 \mathrm{~nm})$, pompé en incidence rasante, et fonctionnant pour le moment en configuration noninjectée (Amplification de l'émission spontanée). Nous espérons qu'une telle étude, menée de manière exhaustive puisse donner des informations sur la meilleure façon d'obtenir des durées d'impulsions plus courtes en régime injecté. D'autre part, la connaissance du profil spectral de la raie laser XUV est cruciale en régime injecté pour déterminer le couplage entre l'harmonique et l'amplificateur.

la finesse de la raie laser $\mathrm{X}\left(\Delta \lambda / \lambda<10^{-4}\right)$ rend difficile sa mesure par spectroscopie classique. Nous avons déduit la largeur spectrale de la source de la mesure de sa cohérence temporelle. Nous avons à cette fin réalisé une expérience d'interférométrie au cours de laquelle nous avons mesuré le contraste de franges d'interférence en fonction de la différence de marche entre les faisceaux qui interférent. Jusqu'ici peu d'études sur la cohérence temporelle des lasers XUV ont été réalisées. Il faut en effet un interféromètre XUV d'excellente qualité utilisant des technologies de pointe. Les premières expériences utilisaient ainsi des interféromètres à division d'amplitude [4,5] équipés de lames séparatrices multicouches XUV. La faible stabilité des lasers XUV et le faible taux de répétition des installations rendaient de plus difficiles la réalisation de séries de mesures de bonne qualité et a fortiori la réalisation d'études de la largeur spectrale en fonction d'un paramètre. 
Dans cette communication nous présentons la première étude systématique de la largeur spectrale d'un laser $\mathrm{X}$ en fonction de ces paramètres de pompage. Nous avons étudié le laser développé sur la station laserix. Il s'agit d'un laser X à Molybdène nickellö̈de $(\lambda=18.9 \mathrm{~nm})$, pompé en incidence rasante et fonctionnant en régime transitoire non-injecté. Cette étude fut possible grâce à l'utilisation d'un interféromètre à division de front d'onde développé par le LIXAM et le LCFIO [6,7], grâce à la bonne stabilité de l'installation LASERIX et à son taux de répétition de $10 \mathrm{~Hz}$, ainsi qu'aux diagnostics utilisés pour contrôler la reproductibilité de l'émission de la source.

\section{DISPOSITIF EXPÉRIMENTAL}

Le laser X étudié à été généré à partir des premiers étages lasers de la station LASERIX. Il s'agit d'un système multi-térawatt, Titane-saphir, de $800 \mathrm{~nm}$ de longueur d'onde centrale et ayant une cadence de $10 \mathrm{~Hz}$. Il délivre des impulsions de $2 \mathrm{~J}$ à 500 ps de durée. Une lame séparatrice installée juste à la sortie de faisceau permet de partager celui-ci en deux sous-faisceaux. Le premier est focalisé directement sur la cible de Molybdène solide à l'aide d'une combinaison de lentilles cylindrique et sphérique suivant une ligne focale de $4 \mathrm{~mm} \times 80 \mu \mathrm{m}$. Cette impulsion a une énergie typique $E_{n s}=500 \mathrm{~mJ}$ et une intensité de $3.10^{11} \mathrm{~W} / \mathrm{cm}^{2}$ sur cible. Elle génère le plasma du laser XUV. La seconde impulsion a une énergie de $E_{p s}=800 \mathrm{~mJ}$ et une durée $\tau_{p s}$ de quelques picosecondes (réglable) après son passage dans un compresseur optique haute énergie. Elle est focalisée sur la cible à l'aide d'un miroir sphérique de $50 \mathrm{~cm}$ de distance focale. La ligne focale d'astigmatisme générée par ce miroir sur la cible est de $4 \mathrm{~mm}$ par $50 \mu \mathrm{m}$. L'intensité obtenue après focalisation est de l'ordre de $10^{14} \mathrm{~W} / \mathrm{cm}^{2}$. L'angle de rasance de cette impulsion avec la cible (angle GRIP [8]) est de $20^{\circ}$. Le délai optimal $\Delta t$ entre les deux impulsions est de 400 ps. Différents diagnostics ont été utilisés pour optimiser le laser XUV ainsi produit et caractériser sa stabilité. Des séries d'images en champ proche et en champ lointain ont été réalisées avant chaque série de mesures interférométriques. Les images en champ proche ont été obtenues à l'aide d'un miroir multicouche asphérisé de $500 \mathrm{~mm}$ de focale formant l'image de la pupille de sortie sur une caméra CCD XUV avec un grandissement de 13. Ce diagnostic donne accès au nombre de photons émis par chaque tir, à la surface et à la forme de la pupille de sortie du laser. Le champ lointain a été obtenu en enregistrant des empreintes de faisceaux avec une caméra CCD placée à $1 \mathrm{~m}$ de la source, derrière un miroir plan multicouche fonctionnant à $45^{\circ}$ d'incidence. Les images en champ lointain facilitent l'optimisation de la source et donnent des informations sur la répartition des non uniformités du faisceau ainsi que sur son pointé.

L'étude de la cohérence temporelle du laser XUV à été réalisée avec un interféromètre à division de front d'ondes représenté sur la figure 1a. Il a été réalisé en collaboration avec le groupe "physique des images" du laboratoire Charles Fabry de l'Institut d'optique LCFIO. Il est constitué d'optiques présentant un excellent état de surface et une bonne réflectivité au rayonnement XUV. Contrairement aux interféromètres XUV à séparatrices, il est par ailleurs achromatique. La différence de marche entre les faisceaux qui interférent peut être modifiée en translatant l'un des dièdres constituant l'interféromètre. En suivant l'evolution du contraste des franges en fonction de la différence de marche introduite il est possible de mesurer la cohérence temporelle de la source. La structure en dièdre (figure 1(b)) permet de garder des conditions de cohérence spatiale constantes lorsque la différence de marche est modifiée.

\section{RÉSULTATS EXPÉRIMENTAUX}

La différence de marche (ddm) nulle a été réglée précisément avant l'expérience à l'aide d'une source à large spectre (le laser infrarouge femtoseconde). La différence de marche entre les deux sous-faisceaux a été changée de 0 à $50000 \lambda$ par pas de $5000 \lambda$. Pour chaque ddm, on a effectué une série de 10 acquisitions en régime mono coup, pour étudier les fluctuations de contraste tir à tir. La visibilité des franges peut fluctuer pour différentes raisons. Premièrement la distribution spatiale de l'intensité du faisceau peut changer tir à tir. Ceci crée une différence d'intensité dans les deux parties du faisceau qui interférent 


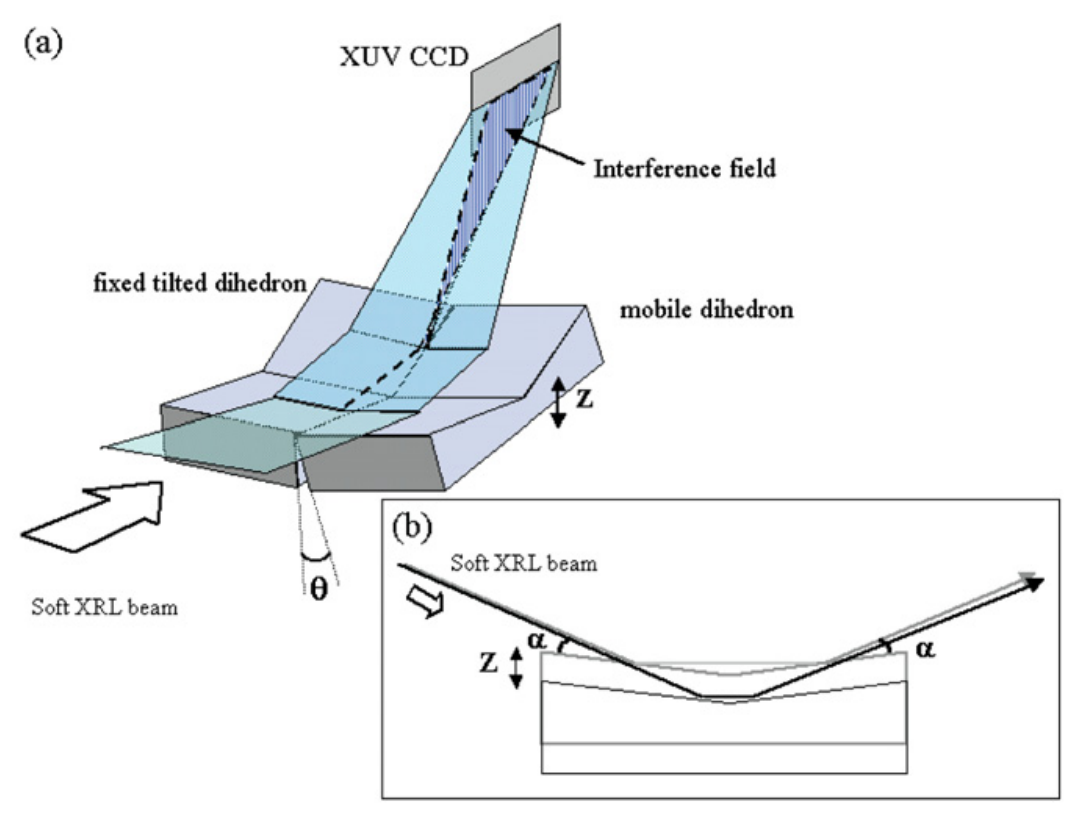

Figure 1. (a) Vue schématique de l'interféromètre à division de front d'onde. (b) Effet de la translation d'un des dièdres sur la marche des faisceaux.

sur la caméra. Deuxièmement, des variations de la taille de la pupille du laser XUV (la source est spatialement incohérente) peuvent créer des fluctuations de cohérence spatiale. Enfin, un troisième point concerne la stabilité du pointé du faisceau. Ces trois sources de fluctuation ont été étudiées et contrôlées en effectuant des acquisitions de champs proches et de champs lointains avant chaque étude en différence de marche. Dans les conditions considérées dans cette étude, ces sources de fluctuation se sont révélées faibles. Nous avons par ailleurs effectués plusieurs mesures de cohérence temporelle dans une même configuration, à des moments différents. Les résultats obtenus sont parfaitement reproductibles.

Nous avons étudié la cohérence temporelle pour différentes configurations de pompage. Nous présentons dans ce qui suit (figures 2) la variation du contraste en fonction des différences de marche pour différents cas expérimentaux. Chaque point sur la courbe représente la moyenne de 10 tirs. Les barres d'erreur indiquent les fluctuations de la visibilité des franges (standard déviation). Les données expérimentales ont été fittées par des fonctions analytiques usuelles. En utilisant le théorème de WienerKhintchine, on peut déterminer la largeur spectrale de la raie en effectuant la transformée de Fourier de la fonction de fit. Dans les cas que nous présentons dans la figure 2, une fonction exponentielle s'avère la plus pertinente, ce qui correspond à un profil Lorentzien. Les figures 2(a) et 2(b) présentent la visibilité des franges en fonction de la différence de marche pour deux énergies de l'impulsion longue $E_{n s}$ différentes. Bien que l'effet soit faible, une diminution de $E_{n s}$ semble induire une diminution de la largeur spectrale. Une plus grande énergie de l'impulsion longue induit peut-être une température ionique dans la zone de gain plus grande et par suite un élargissement Doppler plus important. Des simulations hydrodynamiques doivent être effectuées pour étayer cette interprétation.

Les graphiques des figure 2(c) et 2(d) présentent la variation de la visibilité en fonction de la ddm pour différentes valeurs de la durée de l'impulsion courte $\tau_{p s}=100 \mathrm{fs}$, et $\tau_{p s}=4$ ps avec $E_{n s}=500 \mathrm{~mJ}$. L'effet de variation de la durée de l'impulsion courte apparaît clairement sur la largeur spectrale. On remarque que $\Delta \lambda$ diminue lorsque $\tau_{p s}$ augmente. Il est, à ce stade, difficile de conclure quant à l'origine de cette évolution, mais elle pourrait être liée à l'intensité de l'impulsion courte qui diminue lorsque sa durée augmente. 

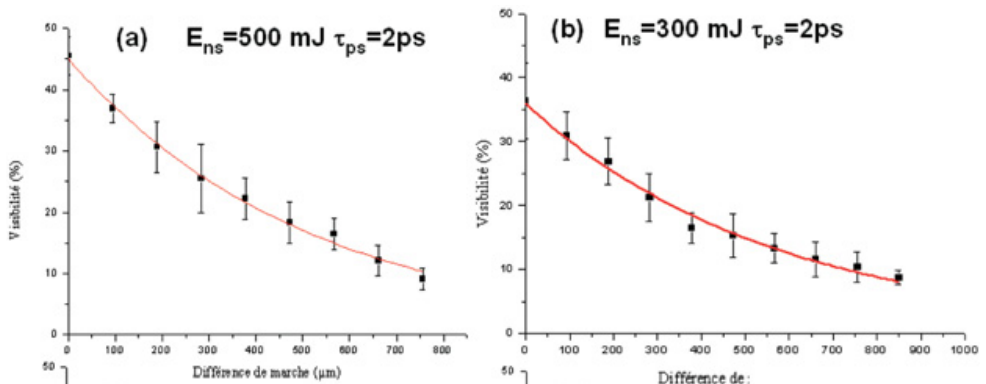

(c) $E_{n s}=500 \mathrm{~mJ} \tau_{\mathrm{ps}}=100 \mathrm{fs}$

(d) $E_{n s}=500 \mathrm{~mJ} \tau_{p s}=4 \mathrm{ps}$
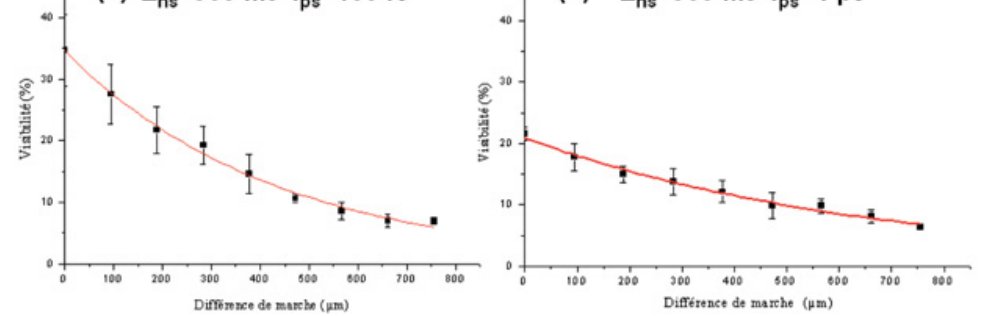

Figure 2. Évolution du contraste des franges en fonction de la différence de marche. (a) l'énergie de l'impulsion longue est $E_{n s}=500 \mathrm{~mJ}$ et la durée de l'impulsion courte de $\tau_{s p}=2 \mathrm{ps}$. (b) $E_{n s}=300 \mathrm{~mJ}$ et $\tau_{s p}=2 \mathrm{ps}$. (c) $E_{n s}=$ $500 \mathrm{~mJ}$ et $\tau_{s p}=100 \mathrm{fs}$. (d) $E_{n s}=500 \mathrm{~mJ}$ et $\tau_{s p}=4 \mathrm{ps}$.

\section{CONCLUSION}

Nous présentons dans cette communication la première étude systématique de la largeur spectrale d'un laser $\mathrm{X}$ en fonction des paramètres de pompe. Cette étude a été rendue possible par la haute cadence de la source et par sa grande stabilité. Nous avons présenté ici l'effet de l'énergie de l'impulsion longue sur la largeur spectrale ainsi que l'effet de la durée de l'impulsion courte. Les variations relatives observées restent faibles mais sont néanmoins significatives. D'autres études visant à étudier l'influence d'une pré-impulsion arrivant avant l'impulsion longue sont en cours d'analyse. Dans tous les cas, l'interprétation des tendances observées nécessitera une modélisation du plasma laser X à l'aide d'un code hydrodynamique. Une telle étude permettra de dégager des stratégies pour élargir la raie laser X et ainsi espérer diminuer sa durée d'impulsion en régime injecté. Cette stratégie devra finalement être confirmée en mesurant directement la largeur spectrale du laser X injecté.

\section{Remerciements}

Nous remercions chaleureusement Jérôme Guigand, Eric Bouisset et Sylvie Appruseze du LIXAM, Jean-Lou Charles, Denis Douillet, Thierry Lefrou du LOA ainsi que tout le personnel administratif du LOA pour leur aide précieuse.

\section{Références}

[1] P. Zeitoun, G. Faivre, S. Sebban, T. Mocek, A. Hallou, M. Fajardo, D. Aubert, P. Balcou, F. Burgy, D. Douillet, et al., Nature 431, 466 (2004).

[2] A. Ayvatyan et al., Eur. Phys. J. D, 20 (2002) 149.

[3] J-P. Goddet, dans cette conférence.

[4] P. Celliers;F. Weber; L.B. Da Silva; T. W. Barbee Jr; R. Cauble; A.S. Wan; J.C. Moreno Optics Letters, Vol. 20 Issue 18, pp.1907-1909 (1995) 
[5] R.F. Smith, J. Dunn, J. Nilsen, J.R. Hunter, S. Hubert, S. Jacquemot, C. Raimond, R. Marmoret, M. Fajardo, Ph. Zeitoun, L. Vanbostal, C.L.S. Lewis, M.F. Ravet and F. Delmotte, Opt. Lett. 28, (2003) 1 .

[6] O. Guilbaud, A. Klisnick, D. Joyeux, D. Benredjem, K. Cassou, S. Kazamias, D. Ros, D. Phalippou, G. Jamelot, and C. Moller, Eur. Phys. J. D 40, 125 (2006).

[7] D. Joyeux R. Mercier, D. Phalippou, M. Mullot, M. Lamare., Proceedings of the 7th international conference on X-ray lasers (EDP Sciences, Paris, 2000), 11511.

[8] K. Cassou, S. Kazamias, D. Ros, F. Plé, G. Jamelot and A. Klisnick, O. Lundh, F. Lindau, A. Persson and C. G. Wahlström, S. de Rossi and D. Joyeux, B. Zielbauer, D. Ursescu and T. Kuehl Optics Letters 32 (2006) 139-41 